\title{
Otimização dos processos de moagem e secagem do pó de alumina/TiC obtido por moagem reativa
}

\section{(Optimization of the milling and drying processes of alumina/TiC powder obtained by reactive milling)}

\author{
V. Trombini ${ }^{1}$, A. H. A. Bressiani ${ }^{1}$, E. M. J.A.Pallone ${ }^{2}$, R. Tomasi ${ }^{3}$ \\ ${ }^{I}$ Centro de Ciência e Tecnologia de Materiais, IPEN, Cidade Universitária, S. Paulo, SP, Brasil \\ ${ }^{2}$ Faculdade de Zootecnia e Engenharia de Alimentos, Pirassununga, USP, SP, Brasil \\ ${ }^{3}$ Departamento de Engenharia de Materiais, UFSCar, S. Carlos, SP, Brasil \\ vthernandes@ipen.br,abressia@ipen.br,eliria@usp.br,tomasi@power.ufscar.br
}

\begin{abstract}
Resumo
A presença de aglomerados em pós cerâmicos é fonte conhecida de heterogeneidade e defeitos nos produtos finais, uma vez que na etapa de sinterização ocorre densificação diferencial dos aglomerados. Assim, o controle de todas as etapas do processamento para obtenção de corpos cerâmicos livres de aglomerados e com microestrutura homogênea é de fundamental importância. Neste trabalho foram avaliadas as condições mais adequadas para reduzir a presença de agregados proveniente da etapa de moagem de alta energia, bem como o controle da etapa de secagem para a obtenção de uma microestrutura com tamanho de grãos pequenos e distribuição homogênea das partículas de inclusões no interior da matriz.
\end{abstract}

Palavras-chave: alumina, TiC, secagem, moagem de alta energia.

Abstract

Agglomerates in ceramic powders often cause heterogeneities and defects in the final ceramic product, due to differential densification of the agglomerates during the sintering stage. Hence, control of the processing steps is important to form ceramic bodies without agglomerates and with a homogeneous microstructure. In this study, the processing conditions to reduce aggregate formation during high-energy milling and control of the powder drying step were evaluated to attain a microstructure consisting of small grains and a homogeneous distribution of TiC inclusions in the matrix.

Keywords: alumina, TiC, drying, high energy milling.

\section{INTRODUÇÃO}

As propriedades finais dos materiais cerâmicos são fortemente afetadas pelo tipo de microestrutura apresentada pelos mesmos, bem como pela presença de defeitos microestruturais. A presença de defeitos e o tipo de microestrutura apresentada pelos materiais cerâmicos são determinados, em grande parte, pela rota de processamento utilizada. Isto torna o controle de todas as etapas do processo de fundamental importância para a obtenção de produtos cerâmicos de maior confiabilidade [1-8].

Uma das grandes dificuldades da utilização de pós ultra-finos para a produção de peças cerâmicas é a alta susceptibilidade destes para a formação de aglomerados durante o processamento, e o efeito destes nas propriedades finais dos produtos cerâmicos. Vários processos vêm sendo estudados com o intuito de diminuir a formação de agregados ou aglomerados fortes tendo-se em vista que a presença destes leva a produção de corpos a verde com densidade heterogênea, prejudicando a sua densificação e gerando produtos com baixa resistência mecânica [1, 2-4]. A formação de aglomerados ocorre de várias maneiras. A força de Van der Waals é a causa inicial da adesão das partículas, sendo muito pronunciada em partículas com alta área superficial específica. Outros mecanismos podem reforçar as ligações entre as partículas, resultando em aglomerados mais resistentes à quebra. $\mathrm{O}$ que determina as características de cada aglomerado é o tipo de ligação envolvida na sua formação. Assim, os mesmos são classificados em agregados ou aglomerados fortes e aglomerados fracos. Os agregados ou aglomerados fortes são assim denominados por possuírem ligações de caráter forte; ou seja, ligações primárias geradas por reações químicas ou sinterização, enquanto que os aglomerados fracos possuem ligações relativamente fracas, como de Van der Waals, eletrostática, magnética ou por capilaridade $[5,6]$.

Estudos sobre o comportamento de alguns pós cerâmicos obtidos por moagem reativa mostram que a produção de agregados é intrínseca ao método de moagem de alta energia com reação auto-sustentada. Esse efeito ocorre devido às altas temperaturas atingidas durante a reação, que podem levar inclusive à fusão parcial dos produtos da reação, induzindo a formação de ligações primárias características dos agregados [9-17]. Moagem é a maneira mais tradicional 
para minimizar a presença de aglomerados no pó cerâmico, sendo a moagem a úmido uma técnica muito eficiente, pois além de reduzir significativamente a presença de aglomerados pode ser utilizada para a mistura de pós. Entretanto após a moagem é necessária a remoção do líquido utilizado no processo, o que muitas vezes pode causar a reaglomeração dos pós, bem como segregação [5]. Com intuito de evitar a formação de agregados durante a etapa de secagem, Sumita e colaboradores [1] estudaram o efeito de vários defloculantes orgânicos em dispersões de aluminas de alta pureza, em meio aquoso. Foi observado que pós de alumina contendo uma pequena quantidade de alumina de transição, quando moídos e desaglomerados em meio aquoso ácido, durante a etapa de secagem reagem formando agregados, os quais não são eliminados nas etapas subseqüentes do processamento. Estudos posteriores mostram que a utilização de meios nãoaquosos, com agente dispersante adequado, por exemplo, etanol e PABA, leva à redução da formação de agregados. Assim, nesse trabalho optou-se pelo uso de etanol nas etapas de desaglomeração e mistura do pó de alumina com as inclusões utilizadas [1].

No presente trabalho foram avaliadas diferentes condições de processamento para a obtenção de nanocompósito de alumina contendo $5 \%$ vol de $\mathrm{TiC}$, com microestrutura homogênea.

\section{PROCEDIMENTO EXPERIMENTAL}

Para a obtenção dos pós de alumina-carbeto de titânio, foi utilizada moagem reativa de alta energia. No processo foram utilizados como reagentes, pós de alumínio metálico (Alcoa, Brasil, 99,7\%), $\mathrm{TiO}_{2}$ (Vetec, 99,5\%) e negro de fumo (Zanini\& Curtis), de acordo com a estequiometria da equação A.

$$
3 \mathrm{TiO}_{2}+4 \mathrm{Al}+3 \mathrm{C} \rightarrow 3 \mathrm{TiC}+2 \mathrm{Al}_{2} \mathrm{O}_{3}
$$

$\mathrm{Na}$ moagem reativa (MR) utilizou-se moinho de alta energia (Spex 8000 mixer/mill), com frascos e bolas de aço temperado. As condições de moagem foram definidas de acordo com trabalhos anteriores [8]. Para o estudo das melhores condições de processamento, com a formação mínima de agregados durante a reação, e redução do tamanho das partículas a dimensões nanométricas, o pó foi moído por mais 1,3 e 5 h após a ocorrência da reação. $\mathrm{O}$ pó obtido foi desaglomerado em moinho planetário (Pulverizette 7 Fritsch $\mathrm{GmbH}$ ) utilizando-se frasco e bolas de aço, em meio alcoólico, com 0,2\% em massa de defloculante, 4-acido aminobenzóico (PABA, Fluka), durante 1 h. Após a desaglomeração o pó foi submetido à lixívia ácida em solução de $20 \%$ de $\mathrm{HCl}$, seguida de lavagem em água destilada, para a retirada do ferro proveniente dos meios de moagem. Para a caracterização e determinação do tamanho médio de cristalito dos materiais formados, foi utilizada a técnica de difração de raios X (difratômetro Siemens 5100); o tamanho médio de cristalito foi calculado por meio da medida do alargamento dos picos de difração com a fórmula de Scherrer; o pó foi caracterizado também pela técnica de microscopia eletrônica de transmissão (Philips CM120) em amostras obtidas pela dispersão do material sobre suporte de cobre, com recobrimento de carbono, e pela técnica de área de superfície específica (BET Gemini 2370, Micromeritics). Agregados grandes formados durante a reação foram embutidos em resina, polidos e analisados por microscopia eletrônica de varredura (Philips XL30-FEG).

O pó de alumina/carbeto de titânio obtido por MR foi misturado a alumina de alta pureza, AKP-53 Sumitomo (99,995\% de pureza e tamanho médio de partícula 0,2 $\mu \mathrm{m})$, de modo a resultar num teor global de $5 \%$ em massa de inclusões nanométricas de TiC. A mistura dos pós foi feita em moinho de bolas, por $14 \mathrm{~h}$, em solução alcoólica com $0,2 \%$ em massa de PABA, 0,5\% em massa de ácido oléico e 100 ppm de MgO. Para a secagem da suspensão alcoólica foram utilizadas três diferentes rotas: secagem sob fluxo de ar, ainda no interior do frasco de moagem com agitação manual constante por $\sim 10 \mathrm{~h}$, secagem em mini spray dryer (Buchi) e secagem em placa de gesso em temperatura ambiente.

Os pós obtidos foram conformados na forma de pastilhas de $10 \mathrm{~mm}$ de diâmetro e $\sim 5 \mathrm{~mm}$ de altura por prensagem uniaxial, $50 \mathrm{MPa}$, seguida de prensagem isostática a $200 \mathrm{MPa}$, e sinterizados a $1500^{\circ} \mathrm{C}$ por $1 \mathrm{~h}$, sob alto vácuo $\left(\sim 10^{-5} \mathrm{mbar}\right)$, forno Thermal Technology Inc. com elemento resistivo de tungstênio. As amostras obtidas foram avaliadas por meio de medida de densidade aparente pelo método de Archimedes e por microscopia eletrônica de varredura.

\section{RESULTADOS E DISCUSSÃO}

A Fig. 1 apresenta o difratograma de raios $\mathrm{X}$ dos produtos da reação $\mathrm{A}$, onde podemos observar a presença apenas dos produtos de reação esperados.

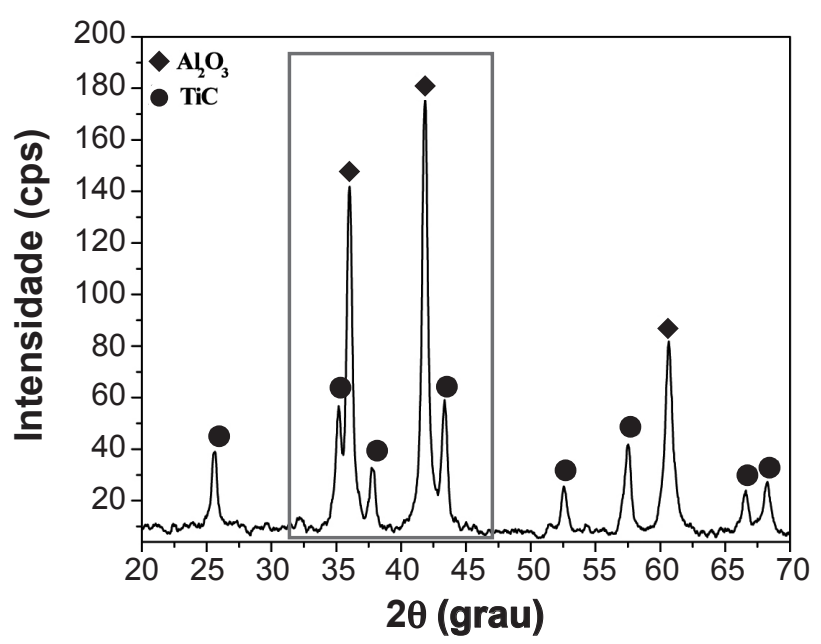

Figura 1: Difratograma de raios $\mathrm{X}$ dos produtos da reação (A) $3 \mathrm{TiO}_{2}+4 \mathrm{Al}+3 \mathrm{C} \rightarrow 3 \mathrm{TiC}+2 \mathrm{Al}_{2} \mathrm{O}_{3}$, com destaque na região utilizada para o cálculo do tamanho de cristalito utilizando a fórmula de Scherrer.

[Figure 1: $X$-ray diffraction pattern of the reaction products $(A)$ $3 \mathrm{TiO}_{2}+4 \mathrm{Al}+3 \mathrm{C} \rightarrow 3 \mathrm{TiC}+2 \mathrm{Al}_{2} \mathrm{O}_{3}$; the detail shows the region used to calculate crystallite size using the Scherrer formula.] 
Para a determinação da melhor condição de processamento dos pós obtidos por moagem reativa foi feita a caracterização dos agregados provenientes da moagem com reação. A Fig. 2 apresenta a micrografia obtida por MEV de seção polida do agregado formado durante a reação, sendo possível observar a presença de duas fases distintas, sendo a região mais escura a alumina e a região mais clara o carbeto de titânio, observa-se também que as fases presentes estão fortemente ligadas devido às altas temperaturas atingidas durante a reação. A Fig. 3 apresenta micrografia de MET e a difração de elétrons de área selecionada (SAD) de agregados de alumina/TiC formados durante a reação, nessa figura pode-se observar que apesar de aglomerado o pó obtido por moagem reativa é composto por partículas nanométricas cristalinas com alta concentração de defeitos, as quais podem ser separadas. Visando a quebra dos agregados, Figs. 2 e 3, o pó formado foi moído por $1 \mathrm{~h}$ após reação, ainda no interior do moinho de alta energia. A moagem em moinho de alta energia utilizando moinho Spex é muito eficiente na quebra de partículas duras, porém devido à alta energia envolvida no processo é inevitável a formação de aglomerados, proveniente de uma compactação das partículas finas dos

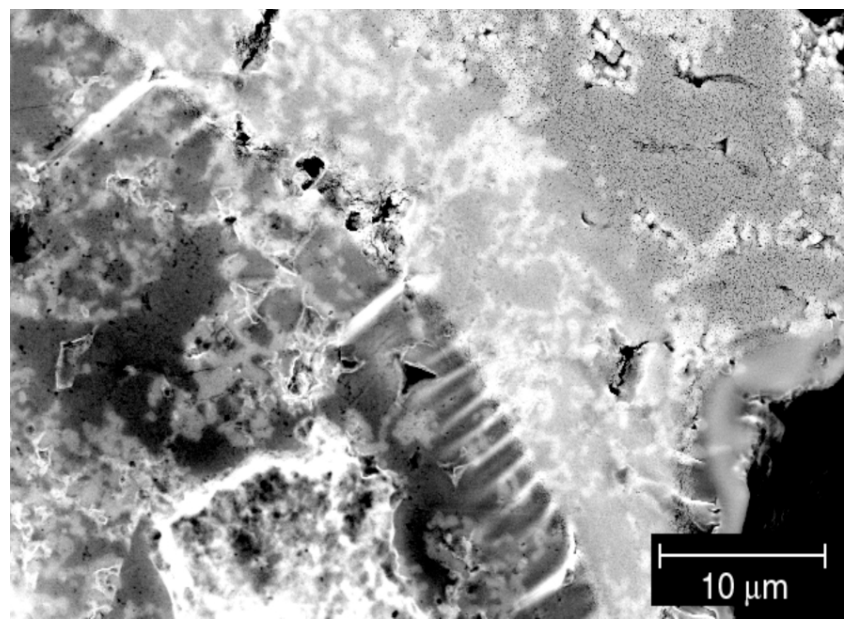

Figura 2: Micrografia obtida por MEV da secção polida do agregado formado durante a reação.

[Figure 2: SEM micrograph of polished section of aggregate formed during reaction.]

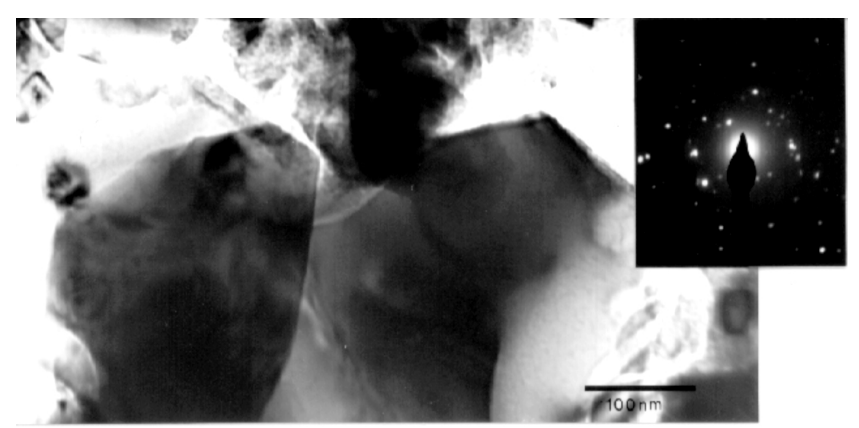

Figura 3: Micrografia obtida por MET utilizando campo claro, do pó de alumina/TiC obtido logo após a ocorrência da reação.

[Figure 3: TEM micrograph of alumina/TiC powder obtained just after reaction occurrence.] pós, sendo necessária a desaglomeração dos pós obtidos em moinho Spex em moinho planetário em meio alcoólico.

Na Fig. 4a é apresentada a micrografia do pó de alumina/ TiC, sem moagem em moinho de alta energia após reação e (b) do pó de alumina/TiC moído em moinho de alta energia durante $1 \mathrm{~h}$ após a ocorrência da reação. Nos dois casos os pós foram submetidos a etapa de desaglomeração em moinho planetário. Como pode ser observado, a moagem de $1 \mathrm{~h}$ do pó de alumina/TiC em moinho Spex, seguida de desaglomeração em moinho planetário, proporciona uma diminuição significativa do tamanho das partículas, porém ainda é possível observar a presença de alguns aglomerados grandes, não eliminados nos processos de moagem e desaglomeração.
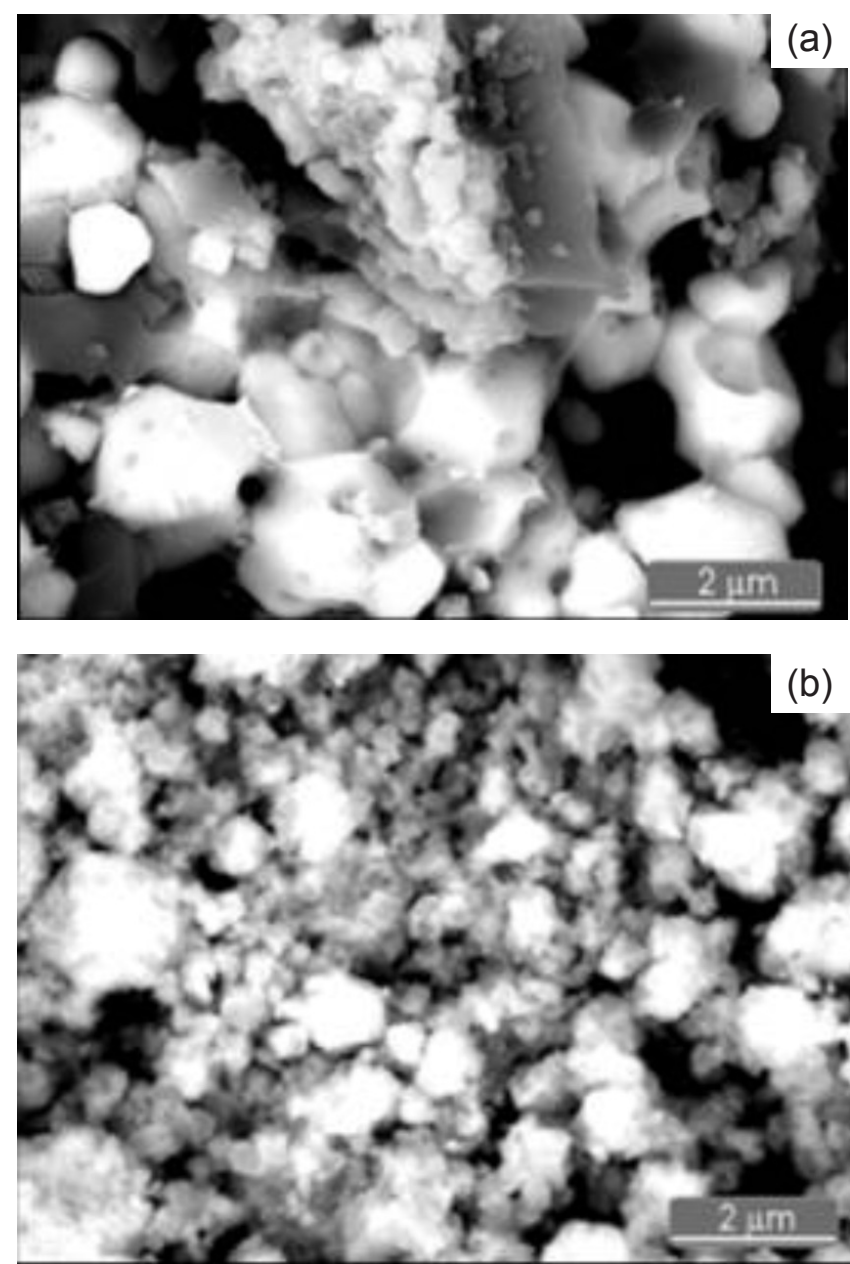

Figura 4: Micrografia obtida por MEV do pó de alumina/TiC (a) sem moagem em moinho de alta energia logo após a reação e (b) moído em moinho de alta energia durante $1 \mathrm{~h}$ após a ocorrência da reação após $1 \mathrm{~h}$ de moagem.

[Figure 4: SEM micrograph of alumina/TiC powder (a) without milling in high energy mill just after reaction and (b) milled during after $1 \mathrm{~h}$ of milling.]

O pó de alumina TiC moído por $1 \mathrm{~h}$ em moinho de alta energia e desaglomerado em moinho planetário foi misturado com a alumina para obtenção de nanocompósitos com teor global de 5\% massa de inclusões de TiC. Amostras 
da mistura foram sinterizadas a $1500{ }^{\circ} \mathrm{C}$, por $1 \mathrm{~h}$, em alto vácuo. A Fig. 5 apresenta uma micrografia da amostra polida, podendo-se observar agregados provenientes das etapas anteriores do processo. Devido à presença dos agregados a amostra $\mathrm{Al}_{2} \mathrm{O}_{3} / \mathrm{TiC}$ apresenta regiões densas com alto teor de alumina (cinza) e inclusões finas de TiC (pontos claros), cercadas por regiões de menor densidade com inclusões de $\mathrm{TiC}$ de maior tamanho. A maior porosidade dessas regiões foi causada pela maior concentração e maior tamanho das inclusões de TiC.

As partícula grandes de $\mathrm{TiC}$, observadas na micrografia da Fig. 5, podem ser agregados remanescentes da etapa de obtenção das partículas por moagem de alta energia, bem como agregados formados pela má distribuição de partículas na fase de mistura e secagem em estufa, da suspensão alcoólica. Na etapa de secagem pode ocorrer a segregação das partículas de TiC, que são mais densas que as partículas de alumina. Esses resultados motivaram a realização de estudos com diferentes tempos de moagem após reação e diferentes procedimentos de secagem.

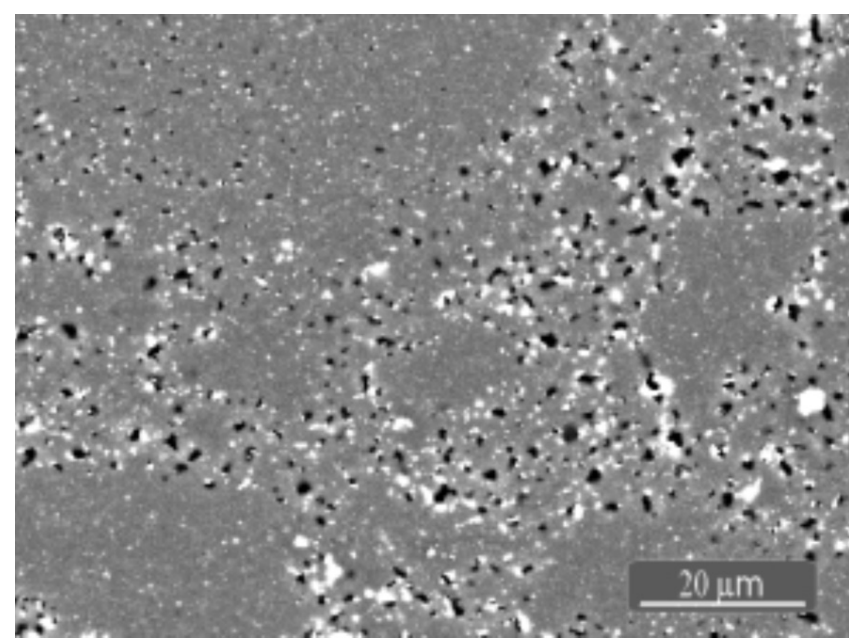

Figura 5: Micrografia obtida por $\mathrm{MEV}$ da amostra de $\mathrm{Al}_{2} \mathrm{O}_{3}$ contendo $5 \%$ peso de TiC obtido por moagem de alta energia por $1 \mathrm{~h}$ e desaglomerado em moinho planetário, sinterizada a $1500{ }^{\circ} \mathrm{C}$ por $1 \mathrm{~h}$ em atmosfera de alto vácuo ( $89 \% \mathrm{DT})$.

[Figure 5: SEM micrograph of alumina containing 5\%wt of TiC, sintered at $1500{ }^{\circ} \mathrm{C}$ during $1 \mathrm{~h}$ in high vacuum atmosphere (89\%TD).]

Para a minimização da presença de agregados de partículas nanométricas de TiC os pós obtidos por moagem reativa foram moídos por maiores tempos em moinho de alta energia (Spex).

A Tabela I apresenta os valores de tamanho de cristalito determinados por difratometria de raios $\mathrm{X}$ utilizando a fórmula de Scherrer Fig. 1, área superficial específica, obtida através do método de BET, e tamanho médio de partículas, calculado a partir dos valores de área superficial específica, para os pós obtidos por moagem com reação moídos em moinho de alta energia durante 3 e 5 h após reação e desaglomerados em moinho planetário por $1 \mathrm{~h}$. Pode-se observar uma diferença significativa nos valores obtidos de
Tabela I - Tamanho médio de cristalito determinado por difração de raios $\mathrm{X}$, área de superfície e tamanho médio de partícula, dos pós obtidos por moagem reativa.

[Table I - Average crystallite size determinate by XRD, specific surface area and average particle size of the powders obtained by reactive milling.]

\begin{tabular}{cccc}
\hline \multicolumn{3}{c}{ Pó de alumina TiC obtido por moagem reativa } \\
\hline $\begin{array}{c}\text { Tempo de } \\
\text { moagem } \\
(\mathrm{h})\end{array}$ & $\begin{array}{c}\text { Tamanho } \\
\text { de cristalito } \\
(\mathrm{nm})\end{array}$ & $\begin{array}{c}\text { Área de } \\
\text { superfície } \\
\text { específica } \\
\left(\mathrm{m}^{2} / \mathrm{g}\right)\end{array}$ & $\begin{array}{c}\text { Tamanho } \\
\text { médio de } \\
\text { partícula } \\
(\mathrm{nm})\end{array}$ \\
\hline 3 & 28,3 & 32,2 & 42,6 \\
5 & 26,6 & 31,5 & 42,5 \\
\hline
\end{tabular}

tamanho médio de partículas e de tamanho de cristalito para os pós de alumina/TiC. Essa diferença é atribuída à presença de aglomerados de cristalitos densos formados por alumina e TiC que não é penetrado pelo gás utilizado no ensaio de área específica superficial. Observa-se também que o maior tempo de moagem não proporciona redução significativa nos valores de tamanho médio de partículas.

As micrografias apresentadas nas Figs. 6a e 6b apresentam as microestruturas das amostras obtidas com a utilização de pós moídos por 3 e $5 \mathrm{~h}$ após a ocorrência da reação, desaglomerados em moinho planetário, misturados com alumina de alta pureza até a proporção de $5 \%$ em massa de TiC, e sinterizadas a $1500{ }^{\circ} \mathrm{C}$ por $1 \mathrm{~h}$, em alto vácuo. Comparando-se a Fig. 5 e as Figs. $6 \mathrm{a}$ e $6 \mathrm{~b}$ pode-se observar uma significativa diminuição da presença de agregados de TiC, como resultado da efetividade na utilização de um maior tempo de moagem após reação. Os resultados apresentados na Tabela I e a observação das Figs. 6a e 6b deixa claro que o tempo de $3 \mathrm{~h}$ de moagem após reação foi suficiente para a redução dos agregados grandes.

Observa-se também que o maior tempo de moagem em moinho de alta energia proporciona a obtenção de amostras com menor tamanho de poros e distribuição mais homogênea. A densidade das amostras foi de $89 \%$, amostras contendo material moído durante $3 \mathrm{~h}$ após reação, e $91 \%$, amostras obtidas com material moído por $5 \mathrm{~h}$ após reação.

Uma vez reduzida a presença de agregados no pó de alumina/TiC obtidos por moagem reativa, a próxima etapa do processo é a determinação do melhor método de secagem da suspensão contendo $5 \%$ em massa de TiC, pois como pode ser observado na Fig. 5 o método de secagem em estufa não se mostrou uma técnica adequada. Assim foram testadas três diferentes técnicas, secagem em spray-dryer, sob agitação em moinho de bolas com o auxílio de fluxo de ar, e em placa de gesso. As Figs. 7a, 7b e 7c apresentam respectivamente as micrografias das amostras sinterizadas produzidas a partir dos pós secos pelos três diferentes métodos, onde pode ser observado que as mesmas não apresentaram o problema de segregação observado na amostra produzida utilizando o pó seco em estufa. As amostras estão homogêneas com boa dispersão das inclusões de TiC. 



Figura 6: Micrografias das amostras de alumina contendo 5\%peso TiC sinterizadas a $1500{ }^{\circ} \mathrm{C}$ por $1 \mathrm{~h}$ em alto vácuo. As inclusões de TiC foram moídas em moinho de alta energia por (a) 3 h (89\%DT) e (b) $5 \mathrm{~h}$ após a reação (91\%).

[Figure 6: Micrographs of alumina samples containing 5wt.\% of TiC sintered at $1500^{\circ} \mathrm{C}$ during $1 \mathrm{~h}$ in high vacuum. Inclusions of $\mathrm{TiC}$ were milled in high-energy mill during (a) $3 \mathrm{~h}$ (89\%TD) and (b) 5 h after the reaction (91\%TD).]

As densidades aparentes obtidas para as amostras produzidas a partir dos pós secos utilizando diferentes tipos de secagem foram de $90 \% \mathrm{DT}$, $89 \% \mathrm{DT}$ e $92 \% \mathrm{DT}$, para as amostras obtidas por secagem em spray-dryer, secagem em moinho de bolas e secagem em placa de gesso, respectivamente. Apesar da pequena diferença nos valores de densidade, observa-se que a microestrutura da amostra produzida utilizando o pó seco em spraydryer apresenta poros maiores quando comparada com as amostras produzidas utilizando os pós secos em moinho e em placa de gesso. Esses resultados podem ser explicados pela formação de aglomerados durante a secagem em spraydryer, que não foram quebrados durante a conformação. Esses aglomerados, após a queima, levam ao aparecimento de regiões de maior densidade e regiões porosas, com poros grandes. Os dois métodos de secagem, em moinho e na placa de gesso mostraram-se eficientes, e uma vez que o método de secagem no interior do moinho é mais fácil e proporciona menor perda de pó este será utilizado.
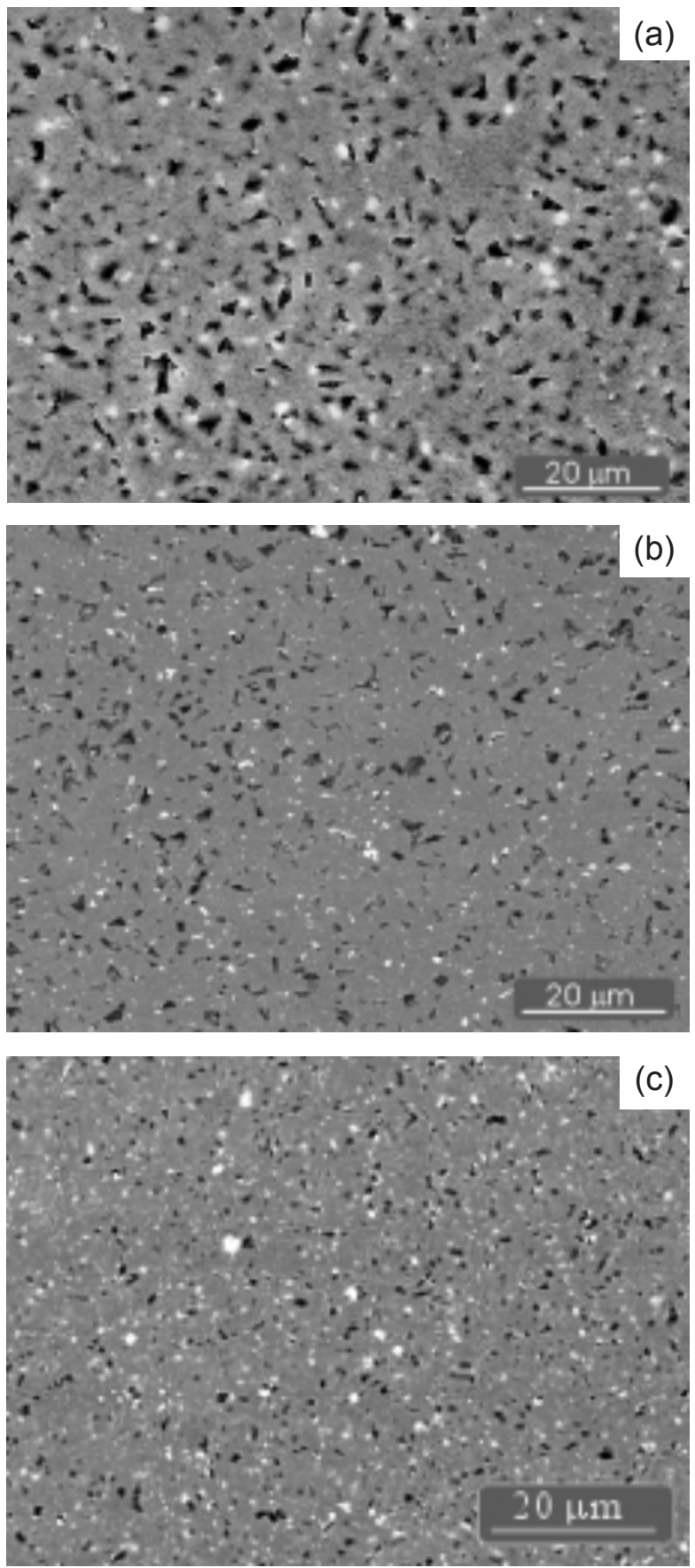

Figura 7: Micrografias das amostras de alumina contendo $5 \%$ peso de $\mathrm{TiC}$, sinterizada a $1500{ }^{\circ} \mathrm{C}$ por $1 \mathrm{~h}$, obtidas por diferentes procedimentos de secagem: (a) secagem em "spray-dryer", (b) secagem sob agitação dentro do moinho de bola com o auxílio de um fluxo de ar e (c) secagem em placa de gesso.

[Figure 7: SEM micrographs of alumina containing 5wt.\% of TiC sample sintered at $1500{ }^{\circ} \mathrm{C}$ during $1 \mathrm{~h}$, obtained by different drying methods: (a) spray-dryer, (b) inside ball mill with air flux and (c) gypsum plate drying.]

\section{CONCLUSÕES}

$\mathrm{O}$ método de moagem reativa mostrou-se eficiente na obtenção de pós nanométricos de alumina/carbeto de titânio, 
com a a formação de agregados, extremamente prejudiciais às etapas subsequentes do processamento. A moagem por $3 \mathrm{~h}$ em moinho de alta energia, após a reação, foi eficiente na quebra desses agregados, mas causou a formação de aglomerados, sendo necessária a desaglomeração do pó em moinho tipo planetário por $1 \mathrm{~h}$. A utilização dos diferentes métodos de secagem tornou possível a obtenção de microestrutura com distribuição homogênea das partículas de carbeto de titânio na matriz de alumina, sendo o método de secagem sob fluxo de ar, o mais prático, pois não necessita de equipamentos sofisticados.

\section{REFERÊNCIAS}

[1] S. Sumita, W. E. Rhine, K. Bowen, J. Am. Ceram. Soc. 74, 9 (1991) 2189.

[2] M. Kityama, J. A. Pask, J. Am. Ceram. Soc. 79, 8 (1996) 2003.

[3] H. Ferkel, R. J. Hellmig, Nanostructured Mater. 11, 5 (1999) 617.

[4] R. Tomasi, A. A. Rabelo, A. S. A. Chinellato, L. Reis, W. J. Botta F., Cerâmica 44, 289 (1998) 170.

[5] J. S. Reed, Principles of Ceramics Processing, $2^{\text {nd }}$ Ed., John Wiley \& Sons, New York, EUA (1995).

[6] S. J. Milne, Mater. Sci. Eng. A 130 (1990) 263.
[7] D. Sen, S. Mazumder, J. S. Melo, Arshad Khan, S. Bhattyacharya, S. F. D’Souza, Langmuir 25, 12 (2009) 6690. [8] J. M. Córdoba, M. J. Sayague, M. D. Alcalá, F. J. Gotor, J. Am. Ceram. Soc. 90, 2 (2007) 381.

[9] L. Takacs, Int. J. Self-Propagating High-Temp. Synt. 18, 4 (2009) 276.

[10] A. P. Nakajato, E. M. J. A. Pallone, A. S. A. Chinelatto, W. J. Botta F., R. Tomasi, Anais 13ํㅡㄹ. Cong. Bras. Ciência Eng. Materiais, Curitiba, PR (1998) p. 5260.

[11] E. M. J. A. Pallone, R. Tomasi, W. J. Botta F., Mater. Sci. Forum 346 (2000) 393.

[12] E. M. J. A, Pallone, D. E. Hanai, R. Tomasi, W. J. Botta F., Mater. Sci. Forum 269 (1998) 289.

[13] R. Tomasi, E. M. J. A. Pallone, W. J. Botta F., Mater. Sci. Forum 312 (1999) 333.

[14] E. M. J. A. Pallone, A. P. Nakajato, W. J. Botta F., R. Tomasi, Anais $44^{\circ}$ Cong. Bras. Cerâmica, S. Pedro, SP (2000) 10901.

[15] W. J. Botta F., R. Tomasi, E. M. J. A. Pallone, A. R. Yavari, Scripta Mater. 44 (2001) 1735.

[16] E. M. J. A. Pallone, V. Trombini, M. S. Nascimento, W. J. Botta F., R. Tomasi, Anais 45 Cong. Bras. Cerâmica, Florianópolis, SC (2001) 0304701.

[17] E. M. J. A. Pallone, V. Trombini, W. J. Botta F., R. Tomasi, Mater. Sci. Forum 14 (2002) 65.

(Rec. 14/10/2010, Rev. 24/03/2011, Ac. 25/03/2011) 